\title{
Experimental study of emission Z-pinch spectra in the axial and radial directions at the Angara- 5-1 facility
}

\author{
V.V. Alexandrov ${ }^{1}$, G.S. Volkov ${ }^{1}$, E.V. Grabovsky ${ }^{1}$, A.N. Gritsuk ${ }^{1, *}$, K.N. Mitrofanov ${ }^{1}$, \\ G.M. Oleinik ${ }^{1}$, and A.P. Shevelko ${ }^{2}$ \\ ${ }^{1}$ State Research Center of the Russian Federation Troitsk Institute for Innovation and Fusion \\ Research, 108840 Troitsk, Moscow, Russia \\ ${ }^{2}$ P.N. Lebedev Physical Institute RAS, 119991 Moscow, Russia
}

\begin{abstract}
Data on the energy, power and spectra composition of the soft $\mathrm{x}$-ray pulse of powerful Z-pinch plasmas in the axial and radial directions in the photon energy range of $0.02-2 \mathrm{keV}$ are presented. The data are obtained from the analysis of experimental results on the implosion of cylindrical arrays with a diameter of $1.2 \mathrm{~cm}$ and a height of $1.6 \mathrm{~cm}$ of tungsten wires diameter of $6 \mu \mathrm{m}$, the linear mass of $220 \mu \mathrm{g} / \mathrm{cm}$ at a current in the range of 2.2-3.5 MA at the Angara-5-1 facility.
\end{abstract}

Currently, to create a substance of high energy density using a spatial concentration of energy flows generated by powerful sources of energy - energy drivers. One of these drivers is a powerful source of soft X-ray radiation (SXR) on the basis of Z-pinch discharges with the use of multi-module accelerators. The spatial distribution of the plasma density during the process of implosion stagnation Z-pinch has a significant impact on the current distribution, the dissipation of magnetic energy, the duration and power of soft $\mathrm{X}$ rays. Implosion of multiwire array starts at the local depletion of the material, so it is not uniform in the axial direction and in the azimuth plane.

The experiments were performed at the Angara-5-1 facility, which consists of eight parallel modules. The amplitude of the pulse current reaches $3.5 \mathrm{MA}$, maximum voltage at the beginning of the water transmission line is $0.8-0.9 \mathrm{MV}$, voltage pulse width at half maximum of $\sim 100 \mathrm{~ns}$. The power and spatial anisotropy of the pinch SXR emission were measured using two identical sets of four vacuum X-ray detectors (XRDs) on the basis of open vacuum photodiodes with different filters [1]. XUV emission spectra of Z-pinch plasma were recorded using the grazing incidence spectrograph GIS-1 (concave grating with a radius of $R=1 \mathrm{~m}, 600$ grooves $/ \mathrm{mm}, \mathrm{W} / \mathrm{Re}$ coating, grazing angle of $4^{\circ}$ ) in the spectral range of $20-400 \AA$ [2].

In the experiments, the power and energy of Z-pinch radiation were obtained in different spectral ranges using XRD-detectors in the axial and radial directions. Anisotropy obtained for these directions is characteristic for all types of loads. The size of the region emitting soft X-rays was determined by this value of the radiation anisotropy. It was found

* Corresponding author: griar@triniti.ru 
that the size of the emitting region is $\sim 4-5 \mathrm{~mm}$ in the SXR range for cylindrical multiwire arrays at peak X-ray power, which corresponds to approximately two to threefold radial compression of the plasma. The size of the emitting region at the time of stagnation, approximately coincides in the ranges of $20-70 \mathrm{eV}$ and $120-280 \mathrm{eV}$. At the same time the image obtained by the time-integrated pinhole camera indicates the presence of a considerable amount of radiating plasma surrounding the pinch - "trailing" mass [3].

The spectra of the emitting plasma were obtained with a spatial resolution. Using these spectra spatial and spectral parameters of the "trailing" mass were determined. These parameters play an important role in the dynamics of implosions and energy power of the whole process because part of the current can flow through it. This could reduce plasma energy near the axis of the array, which is converted into SXR. It is shown that the presence of a trailing" mass reduces the spectral density of radiation and changes the characteristics of spectrum. Sizes of the radiation region obtained from the spectra with a radial resolution coincide with those obtained by XRD-detectors from the radiation anisotropy and pinhole camera images. Comparison of experimental and simulated spectra of radiation of tungsten Z-pinch was carried out.

The procedure to restore the power spectral distribution in different photon energy ranges was solved using data from two sets of XRD with various filters. It has been shown that the results of measurement of the spectra in the radial direction, obtained by independent methods (from the XRD-detectors and the GIS-1 spectrograph), coincide. In the experiment, it was found that the plasma emission of Z-pinch tungsten multiwire cylindrical array in a radial direction is more "harder" than in the axial direction. That is in a radial direction main part of energy is emitted in the range of $70-300 \mathrm{eV}$, while in the axial direction radiating energy is distributed more evenly between the ranges $20-70$ and $70-300 \mathrm{eV}$.

Thus, the distribution of the SXR Z-Pinch source intensity is relatively isotropic in its height and in the azimuthal angle, and the spectral composition of the radiation in the axial direction corresponds to a "softer" spectrum as compared to the radial.

This work was supported by the Russian Science Foundation under Project № 16-1210487 and in part by the Russian Foundation for Basic Research under Project 15-0204411 .

\section{References}

1. A.V. Branitskiy, G.M. Oleinik, Instrum. Exp. Tech. 22, 480 (2000)

2. A.P. Shevel'ko, D.E. Bliss, E.D. Kazakov, M.G. Mazarakis, J.S. McGurn, L.V. Knight, K.W. Struve, I.Yu. Tolstikhina, T.J. Weeks, Plasma Phys. Rep. 34, 944 (2008)

3. A.N. Gritsuk, V.V. Aleksandrov, E.V. Grabovskiy, Y. Laukhin, K.N. Mitrofanov, G.M. Oleinik, G.S. Volkov, I.N. Frolov, A.P. Shevel'ko, IEEE Transactions on Plasma Science 41, 3184 (2013) 